\title{
Displays and Decisions - The Chicken or the Egg? The Influence of Displays on the Decision Making Process
}

\author{
Patricia L. McDermott \\ Alion Science and Technology \\ pmcdermott@alionscience.com
}

\author{
Michael Barnes \\ Army Research Laboratory \\ michael.j.barnes@us.army.mil
}

\author{
Shaun Hutchins \\ Alion Science and Technology \\ shutchins@alionscience.com
}

\begin{abstract}
Motivation - The goal is to encourage discussion on the philosophical approach to studying decision making. Research approach - A full design cycle included cognitive task analysis (CTA), display design, analysis of alternatives, and redesign. Findings - The visualizations influenced the decision process and the courses of action. Research Implications - This example illustrates an interesting question regarding decision making for envisioned problems and whether the designers should start by understanding how future operators are likely to think about the problem or by designing alternatives and studying their impact on decision making and course of action selection. Originality/Value - Multiple phases of a naturalistic design process are discussed as well as the impact of those displays on decision strategies. Take away message - Even when the design process starts with a CTA, it is important to examine how different ways of presenting or interacting with data effect how operators think about the task and take action.
\end{abstract}

\section{Keywords}

Decision making process, design cycle, visualizations, courses of action.

\section{INTRODUCTION}

This research brings to light an important philosophical question. As Naturalistic Decision Making (NDM) researchers is it our belief that a decision making process exists in a domain and it is our job to discover and support it? Or with the case of an envisioned task, is it our job to create displays and then delve into the decision making process to understand the impact and tradeoffs of displays on the decision process. Or is it some combination of the two? This has similarities to the chicken and the egg problem. In a novel task, which comes first - the decision or the display? The paper provides an example in which the researchers set out to support one decision process, based on a cognitive task analysis, and ended up with different decision processes depending on the visualization. The authors describe a redesign with two different modes of interaction within a single system designed to support two different decision processes both aimed at the same end goal - helping operators determine how to reposition a suite of robotic assets across the battlefield.

Both the information visualization and the mode of interacting with the data can impact decision making. Understanding how displays impact decision process and decision outcome is a critical part of the design of new systems. As tactics, techniques, and procedures are developed, designers can choose the visualization that helps operators think about the problem in a way that is consistent with doctrine.

\section{PHASE I OF THE DESIGN CYCLE: DESIGNING TO SUPPORT THE DECISION:}

Based on a cognitive task analysis with experts at the Battle Command Battle Laboratory at Fort Knox, visualization alternatives were designed to help military operators determine how to reposition robotic assets on the battlefield to conduct reconnaissance. This was a relatively new problem with no existing decision support. The specific tactics, techniques, and procedures had not yet been specified. However, the researchers found that there is often is a singular criteria that assumes the role of a "golden criteria" upon which the decision is primarily based. However, this golden criteria changes depending on the situation. It was stressed that time is often the deciding factor - whatever robotic asset can provide information the quickest will often be the one chosen.

\section{PHASE II OF THE DESIGN CYCLE: UNDERSTANDING THE IMPACT OF DISPLAYS ON DECISION MAKING}

Several display alternatives were developed to support the "golden criteria" framework. Display alternatives were evaluated via empirical data collection coupled with think aloud protocol and cognitive task analysis probes. When used 
by career military experts, the different visualizations resulted in different ways of thinking about the problem and ultimately, different courses of action (McDermott \& Hautamaki, 2007). This represents an example in which the display influenced the decision, particularly in a situation with no established techniques.

\section{PHASE III: DESIGN TO SUPPORT DIFFERENT MODES OF INTERACTION}

In the next iteration of displays focused on supporting two different decision processes. The first is more analytical and the second is designed to support operator expertise. The essence of the Analytical mode is that it presents ten plans and allows operators to interact with the plan criteria in order to understand tradeoffs. For example, the operator could discover that plans that have the highest overall coverage also tend to take the longest amount of time to implement. Therefore, the operator needs to trade off coverage and time. The operator understands the implications of different plans before he or she chooses them.

The Expert Mode takes advantage of an operator's expertise by allowing them to tweak plans. Experts often have knowledge to bear on the situation. This could be a subtle use of the terrain to gain advantage of the enemy, knowledge of the latest enemy tactics, or familiarity with the undocumented strengths and weaknesses of robotic asset types. For example, the operator may know that the enemy forces have been conducting reconnaissance missions at night so the operator makes sure that the robotic assets at the most critical area have night vision capabilities. In the Expert Mode, the system recommends a course of action and the operator can click and drag the assets around the battlefield to reposition them. The operator can then have the system evaluate the plan and compare it to either the suggested plan or other tweaked plans.

These two alternatives represent different ways of interacting with the data. The Expert Mode was developed to support a recognition-primed decision-making process (Klein, 1989). The Analytical mode was developed to support those with less experience because it helps them understand and discover implications of different actions. The modes were not explicitly developed to support different user populations; the purpose was to support different ways of interacting with and learning from the data.

\section{CONCLUSION}

These design examples illustrate several important points regarding the design of new systems. First of all, even when the design process starts with a CTA, the displays themselves have the power to alter and effect the decision making process and therefore change the decision outcomes (i.e., course of action selected). Designers should examine the impact of designs on decision process to ensure that the decision process is being supported as intended. If doctrine has not been specified regarding the "right" was to make the decision (i.e., what data should be considered, whether certain information should be weighted or given priority), this is an opportunity for the researcher to link display alternatives with the associated decision process and let the lessons learned drive the design decisions as the doctrine is developed. As Commanders and policy makers determine which information should be the deciding factor, system developers can choose the best visualizations to support that strategy.

This naturalistic design process illustrates a key philosophical problem. Is it the researcher's job to uncover decision making that exists in the world, or should the researcher seek to design displays the impact of decision making process favorably? Perhaps the best solution is a combination of the two approaches, an iterative design process that includes both study of the decision space independent of displays as well as an examination of how the resulting design or designs impact the decision process.

\section{ACKNOWLEDGMENTS}

Prepared through collaborative participation in the Advanced Decision Architectures Consortium sponsored by the U.S. Army Research Laboratory under the Collaborative Technology Alliance Program, Cooperative Agreement DAAD 1901-2-0009.

\section{REFERENCES}

Klein, G. A. (1989). Recognition-primed decisions. In W. B. Rouse (Ed.), Advances in man-machine systems research, pp. 47-92. Greenwich, CT: JAI Press, Inc.

McDermott, P. L., \& Hautamaki, B. (2007). Resource allocation decisions: The impact of individual differences on preferences and performance. Poster presented at the 8th International Conference on Naturalistic Decision Making, Monterey: CA. 